\title{
Coupled Dynamic Model of Resource Diffusion and Epidemic Spreading in Time-Varying Multiplex Networks
}

\author{
Ping Huang, ${ }^{1,2,3}$ Xiao-Long Chen, ${ }^{4}$ Ming Tang, ${ }^{5,6}$ and Shi-Min Cai ${ }^{1,2,3}$ \\ ${ }^{1}$ School of Computer Science and Engineering, University of Electronic Science and Technology of China, Chengdu 610054, China \\ ${ }^{2}$ Institute of Fundamental and Frontier Sciences, University of Electronic Science and Technology of China, Chengdu 610054, \\ China \\ ${ }^{3}$ Big Data Research Center, University of Electronic Science and Technology of China, Chengdu 610054, China \\ ${ }^{4}$ School of Economic Information Engineering, Southwestern University of Finance and Economics, Chengdu 611130, China \\ ${ }^{5}$ School of Mathematical Sciences, Shanghai Key Laboratory of PMMP, East China Normal University, Shanghai 200241, China \\ ${ }^{6}$ Shanghai Key Laboratory of Multidimensional Information Processing, East China Normal University, Shanghai 200241, China
}

Correspondence should be addressed to Shi-Min Cai; shimin.cai81@gmail.com

Received 27 October 2020; Revised 3 March 2021; Accepted 19 March 2021; Published 28 March 2021

Academic Editor: Ye Wu

Copyright (C) 2021 Ping Huang et al. This is an open access article distributed under the Creative Commons Attribution License, which permits unrestricted use, distribution, and reproduction in any medium, provided the original work is properly cited.

\begin{abstract}
In the real world, individual resources are crucial for patients when epidemics outbreak. Thus, the coupled dynamics of resource diffusion and epidemic spreading have been widely investigated when the recovery of diseases significantly depends on the resources from neighbors in static social networks. However, the social relationships of individuals are time-varying, which affects such coupled dynamics. For that, we propose a coupled resource-epidemic (RNR-SIS) dynamic model (coupled model for short) on a time-varying multiplex network to synchronously simulate the resource diffusion and epidemic spreading in dynamic social networks. The equilibrium analysis of the coupled model is conducted in a general scenario where the resource generation varies between susceptible and infected states and the recovery rate changes between resourceful and noresource states. By using the microscopic Markov chain approach and Monte Carlo simulations, we determine a probabilistic framework of the intralayer and interlayer dynamic processes of the coupled model and obtain the outbreak threshold of epidemic spreading. Meanwhile, the experimental results show the trivially asymmetric interactions between resource diffusion and epidemic spreading. They also indicate that the stronger activity heterogeneity and the larger contact capacity of individuals in the resource layer can more greatly promote resource diffusion, effectively suppressing epidemic spreading. However, these two individual characters in the epidemic layer can cause more resource depletion, which greatly promotes epidemic spreading. Furthermore, we also find that the contact capacity finitely impacts the coupled dynamics of resource diffusion and epidemic spreading.
\end{abstract}

\section{Introduction}

Most real networks are not isolated and the spreading dynamic processes on such real networks described by classical models such as the susceptible-infected-susceptible model (SIS) [1,2] and susceptible-infected-recovery model (SIR) [3] may be interrelated and interactive with each other [4-6]. In epidemic spreading, a variety of factors can greatly influence the spread of diseases, such as the particular individuals' birth rate [7], immunity [8, 9], public policy [10, 11], vaccination [12], awareness [13-19], and resource $[20,21]$.
For example, Brandeau [20] et al. determined the optimal allocation of a limited resource for epidemic control among independent populations, and Chen et al. [21] found that any additional quantity of resources above the critical level would only bring marginal benefits to the containment. These works focus more on how public resource investment affects the spread of disease. However, in a general scenario, many patients tend to depend on the individual resources from their direct or indirect neighbors in social networks because of the limitation of public resource investment. Thus, some researchers naturally investigate the interactions between individual resources and epidemic dynamics. They 
assume that resource diffusion and epidemic spreading occur on a two-layer interdependent network because resources usually propagate through social links and diseases spread through physical contacts. They find that individual resources play a crucial role in controlling or mitigating epidemic spreading by using an appropriate strategy of resource allocation [22-27].

Although many works based on contact network models have been done on the resource allocation for epidemic control, most of them assume that contacts are static through time. However, contact patterns are not static [28-31] and abundant close contacts in heterogeneous social networks promote both epidemic outbreak and spreading [32-35]. In recent years, the activity-driven (AD) temporal network model has attracted a large amount of attention for considering the dynamic connectivity patterns in real networks [19, 36-39]. In such network models, each node is assigned with an activity potential that determines its probability to create pairwise interactions with others randomly selected at each time.

Furthermore, some theoretical methods describe the evolution of the dynamic spreading process and analyze the corresponding stylized characters. Two of the widely used approaches are heterogeneous mean-field (HMF) [40-42] and the microscopic Markov chain approach (MMCA) [43]. MMCA can deal with individual-level infection dynamics much better. This approach is first used to derive the expression of the outbreak threshold of epidemic spreading on epidemic-awareness multiplex networks [44, 45]. Very recently, the MMCA is also expanded to understand the interaction between awareness propagation and epidemic spreading on static multiplex networks $[46,47]$ and timevarying multiplexes $[19,48]$.

In real life, healthy individuals can earn resources (e.g., money) or strive for personal resources. However, patients quickly lose the ability to generate resources. For solving the problem of deficiency of individual resources, patients commonly seek resources from their friends or relatives and even use the crowdfunding platform (e.g., GoFundMe and Waterdrop) to obtain resources provided by healthy individuals. As the resource diffusion and epidemic spreading on dynamic social networks synchronously happen in real society, the present work uses the $\mathrm{AD}$ model to construct a generic time-varying multiplex network representing the active contacts (or social relationships) of social networks. Because the dissemination of resources relies on the spreading of news on the crowdfunding platform, we use the Resourceful-Noresource-Resourceful (RNR) model (i.e., a SIS-like model) to describe resource diffusion. The evolutionary process of epidemic spreading is described by the SIS model. These two dynamic models are coupled into the RNR-SIS model by interlayer links of the time-varying multiplex network better to understand the interactions between resource diffusion and disease spreading. Meanwhile, considering the similarity between awareness propagation and resource diffusion, we make efforts to apply the MMCA for constructing a theoretical framework of the coupled resource-epidemic dynamic model (i.e., coupled RNR-SIS model) on a time-varying multiplex network.
Theoretically, the equilibrium analysis based on MMCA determines a probabilistic framework of the coupled model and theoretical outbreak thresholds involving different parameters. By performing extensive numerical simulations, we obtain the outbreak thresholds of epidemic spreading via variability measurement, which well agree with the theoretical analysis. Moreover, the experimental results also show that stronger activity heterogeneity and larger contact capacity of individuals greatly influence the coupled dynamics of resource diffusion and epidemic spreading. However, the influence of small contact capacity is finite.

Our paper is organized as follows: in Section 2, we describe the coupled RNR-SIS model on a time-varying multiplex network. In Section 3, we use MMCA to propose a probabilistic description of the dynamical processes and derive the theoretical expression of the outbreak threshold. In Section 4, we compare numerical simulations with theoretical analysis and investigate the influences of different parameters on resource diffusion and epidemic spreading. In Section 5, we conclude our work.

\section{Coupled Resource-Epidemic Model on a Time-Varying Multiplex Network}

The coupled RNR-SIS model on a time-varying multiplex network is depicted in Figure 1. In this model, individuals are mapped into the nodes of an $\mathrm{AD}$ temporal two-layer network. The individual resources diffuse on the upper layer (i.e., resource layer), and the spread of disease occurs on the lower layer (i.e., epidemic layer). In the dynamic process of resource diffusion (or epidemic spreading), the nodes are alternatively changing from the noresource (or susceptible) to resourceful (or infected) states according to resource rate diffusion $\lambda$ (or epidemic transmission rate $\beta$ ) and conversely with resource loss rate $\delta$ (or epidemic recovery rate $\mu$ ). $\lambda$ is parallel with $\beta$. For example, the possibility is that you will give a hand when you browse information about fundraising. Thus, in the coupled dynamic process, the nodes are divided into four categories, noresource and susceptible (NS), resourceful and susceptible (RS), noresource and infected (NI), and resourceful and infected (RI). We assume the trivially asymmetric interactions between resource diffusion and epidemic spreading because the individual resources effectively affect the recovery of disease, but the spread of disease only affects the generation of individual resources. Thus, we further refine the epidemic recovery rate into $\mu^{N}$ and $\mu^{R}$ according to the node state in the epidemic layer. Obviously, the infected nodes with the resource can recover faster than those without resources, which indicates $\mu^{N}=\xi \mu^{R}(0 \leq \xi \ll 1)$. Table 1 shows the detailed definition of parameters in the coupled model. Most of the parameters are probabilities and rates, and their domain is limited in the interval $[0,1]$.

More concretely, we construct the AD networks of $N$ nodes for each layer regarding different parameters. Each node $i$ is assigned with an activity level (i.e., firing rate) $a_{i}=\eta_{1} x_{i}$ in the resource layer and $b_{i}=\eta_{2} y_{i}$ in the epidemic layer, where $\eta_{i}$ is a rescaling factor to make the average number of active nodes per unit time be $\eta_{1}\langle x\rangle N$ and 


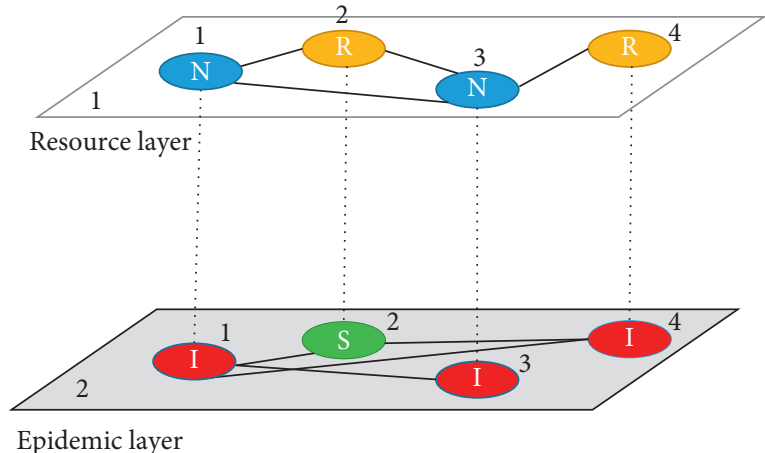

(a)

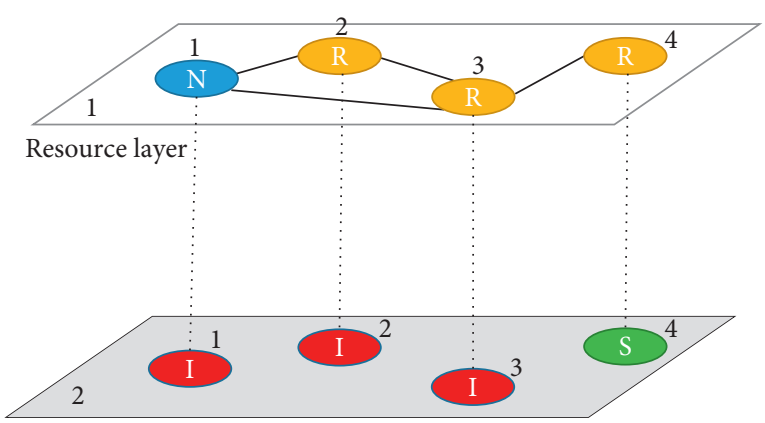

Epidemic layer

(b)

FIGURE 1: The sketch of coupled RNR-SIS model on an AD temporal two-layer network. The nodes indicate individuals in real society, and the edges depict dynamic contacts and social relationships of individuals in social networks. Individual resources diffuse on the upper layer (i.e., resource layer), leading to the node in a resourceful or noresource state. The spread of disease occurs on the lower layer (i.e., epidemic layer), leading to the node in a susceptible or infected state. The coupled dynamic process evolves from (a) times $t_{1}$ to (b) $t_{1}+\Delta t$, where node 2 is infected from its neighbors, node 4 recovers in the epidemic layer, and node 3 changes from the noresource to resourceful state in the resource layer.

TABLE 1: Notions of parameters.

\begin{tabular}{|c|c|c|}
\hline Symbol & Domain & Definition \\
\hline$N$ & {$[0, \infty]$} & Number of nodes in network \\
\hline$t$ & {$[0, \infty]$} & Time step \\
\hline$\delta$ & {$[0,1]$} & Resource loss rate \\
\hline$\lambda$ & {$[0,1]$} & Resource diffusion rate \\
\hline$\beta$ & {$[0,1]$} & Epidemic transmission rate \\
\hline$\mu$ & {$[0,1]$} & Epidemic recovery rate \\
\hline$\mu^{N}$ & {$[0,1]$} & Epidemic recovery rate of the nodes in noresource state \\
\hline$\mu^{R}$ & {$[0,1]$} & Epidemic recovery rate of the nodes in resourceful state \\
\hline$m_{1}$ & {$[0, \infty]$} & Contact capacity of active nodes in the resource layer \\
\hline$m_{2}$ & {$[0, \infty]$} & Contact capacity of active nodes in the epidemic layer \\
\hline$a_{i}$ & {$[0,1]$} & Activity level of node $i$ in the resource layer \\
\hline$b_{i}$ & {$[0,1]$} & Activity level of node $i$ in the epidemic layer \\
\hline$\eta_{1}$ & {$[0, \infty]$} & Rescaling factor of resource layer \\
\hline$\eta_{2}$ & {$[0, \infty]$} & Rescaling factor of epidemic layer \\
\hline$x_{i}$ & {$[\epsilon, 1]$} & Activity potential of node $i$ in the resource layer \\
\hline$y_{i}$ & {$[\epsilon, 1]$} & Activity potential of node $i$ on epidemic layer \\
\hline$\varepsilon$ & $(0,1]$ & The lower cutoff of activity potential \\
\hline$F_{1}(x)$ & - & Distribution of nodes' activity potential of resource layer \\
\hline$F_{2}(y)$ & - & Distribution of nodes' activity potential of epidemic layer \\
\hline$P_{i}^{X}$ & {$[0,1]$} & Probability of node $i$ in state X \\
\hline$r_{i}(t)$ & {$[0,1]$} & Probability that node $i$ has no resources from neighbors at time $t$ \\
\hline$q_{i}(t)$ & {$[0,1]$} & Probability that node $i$ will not be infected by any neighbors at time $t$ \\
\hline$\beta_{c}$ & {$[0,1]$} & The upper threshold of $\beta$ for the outbreak of epidemic \\
\hline$\lambda_{c}$ & {$[0,1]$} & The lower threshold of $\lambda$ for the outbreak of epidemic \\
\hline
\end{tabular}

$\eta_{2}\langle y\rangle N$. According to the previous work [49], the activity levels of individuals in social networks usually obey a power-law distribution with a cutoff, and due to the experience from [36], we use the given probability distributions $F_{1}(x) \propto x^{-\gamma_{1}}$ and $F_{2}(y) \propto y^{-\gamma_{2}}$ to generate the hierarchical activity potential of individuals in the resource and epidemic layer, respectively. Also, we set the lower cutoff $\varepsilon=0.001$ of activity potential, that is, $\varepsilon \leq x_{i} \leq 1$ ( $y_{i}$ is likewise).

Based on the AD temporal two-layer network, we describe the evolving process of coupled RNR-SIS model as the following steps: (i) At each time $t$, node $i(i=1, \ldots, N)$ becomes active with probabilities $a_{i}$ and $b_{i}$ in the corresponding layer. If the node is active in the resource layer, it will generate $m_{1}$ (corresponding $m_{2}$ in the epidemic layer) edges to randomly selected nodes. Otherwise, no connections are created. Both active and inactive nodes can receive connections from active nodes.

(ii) The coupled RNR-SIS dynamic process is run on the constructed two-layer networks. Initially, the nodes only exist in the RS and NI states. The rules are specifically described as follows: (a) only the susceptible nodes can generate resources at the end of 
any moment. (b) To some extent, the resourceful nodes lose their all resources with probability $\delta$ or diffuse resources to their direct neighbors in the noresource state with probability $\lambda$. (c) The susceptible nodes are infected by their direct neighbors in the infected state with probability $\beta$. (d) The infected nodes with (or without) resources recover in the susceptible state with probability $\mu^{R}\left(\right.$ or $\left.\mu^{N}\right)$. Note that the NS nodes only exist at the transient time but finally disappear at the end of the synchronous-updating coupled dynamic process and the resource diffusion to susceptible nodes is insignificant according to rule (a). Thus, there are only three types of nodes in the coupled dynamic process at each time. Figure 2 shows the transition probability trees of three types of nodes describing the transient changes of their states.

(iii) At next time $t+\Delta t$ (we set $\Delta t=1$ ), all edges of the previous network in each layer are deleted and we repeat the construction of $\mathrm{AD}$ networks. The coupled dynamic process continuously evolves according to the rules and also is terminated when it converges to the stable state.

\section{Theoretical Analysis Based on Microscopic Markov Chain Approach}

According to the model mentioned above, each node can be in one of the four states at time $t$ with the probabilities represented by $P_{i}^{\mathrm{NS}}(t), P_{i}^{\mathrm{RS}}(t), P_{i}^{\mathrm{NI}}(t)$, and $P_{i}^{\mathrm{RI}}(t)$. According to rule $(\mathrm{a})$, we can easily obtain $P_{i}^{\mathrm{NS}}(t) \equiv 0$. Note that $P_{i}^{R}=$ $P_{i}^{\mathrm{RS}}+P_{i}^{\mathrm{RI}}$ and $P_{i}^{I}=P_{i}^{\mathrm{NI}}+P_{i}^{\mathrm{RI}}$. Based on MMCA, we give the following theorems.

Theorem 1. $r_{i}(t)$ denotes the probability of node $i$ not getting resources from any direct neighbors, which can be written as

$$
r_{i}(t)=\prod_{j}\left(1-\lambda \frac{m_{1}}{N}\left[a_{i} P_{j}^{R}(t)+a_{j} P_{j}^{R}(t)\right]\right) .
$$

The part $\left(m_{1} / N\right) a_{i} P_{j}^{R}(t)$ is the probability that an active node $i$ creates a link with an active node $j$ in the state $R$ and the second part $\left(m_{1} / N\right) a_{j} P_{j}^{R}(t)$ is the probability that an active node $i$ gets a link from an active node $j$ in the state $R$.

Theorem 2. $q_{i}(t)$ denotes the probability for the active node $i$ not being infected by any neighbors, which can be written as

$$
q_{i}(t)=\prod_{j}\left(1-\beta \frac{m_{2}}{N}\left[b_{i} P_{j}^{I}(t)+b_{j} P_{j}^{I}(t)\right]\right) .
$$

The part $\left(m_{2} / N\right) b_{i} P_{j}^{I}(t)$ is the probability that an active node $i$ creates a link with an active node $j$ in the state $I$ and the second part $\left(m_{2} / N\right) b_{j} P_{j}^{I}(t)$ is the probability that an active node $i$ gets a link from an active node $j$ in the state $I$.
Theorem 3. According to Figure 2, we can develop the Markov chains equations for each node $i$ as follows:

$$
\begin{aligned}
P_{i}^{\mathrm{RS}}(t+1)= & P_{i}^{\mathrm{RS}}(t)\left[\delta q_{i}(t)+(1-\delta) q_{i}(t)\right] \\
& +P_{i}^{\mathrm{NI}}(t)\left[\left(1-r_{i}(t)\right) \mu^{R}+\mu^{N} r_{i}(t)\right] \\
& +P_{i}^{\mathrm{RI}}(t)\left[(1-\delta) \mu^{R}+\delta \mu^{N}\right] \\
P_{i}^{\mathrm{NI}}(t+1)= & P_{i}^{\mathrm{RS}}(t) \delta\left(1-q_{i}(t)\right)+P_{i}^{\mathrm{NI}}(t)\left(1-\mu^{N}\right) r_{i}(t) \\
& +P_{i}^{\mathrm{RI}}(t) \delta\left(1-\mu^{N}\right), \\
P_{i}^{\mathrm{RI}}(t+1)= & P_{i}^{\mathrm{RS}}(1-\delta)\left(1-q_{i}(t)\right) \\
& +P_{i}^{\mathrm{NI}}(t)\left(1-r_{i}(t)\right)\left(1-\mu^{R}\right) \\
& +P_{i}^{\mathrm{RI}}(t)(1-\delta)\left(1-\mu^{R}\right) .
\end{aligned}
$$

The theoretical analysis based on MMCA aims to deduce the theoretical outbreak threshold $\beta_{c}$ of epidemic spreading through theorems (1)-(3). In the stable state, equations (3)-(5) can satisfy $P_{i}^{\mathrm{RS}}(t+1)=P_{i}^{\mathrm{RS}}, P_{i}^{\mathrm{RI}}(t+1)=P_{i}^{\mathrm{RI}}$, and $P_{i}^{\mathrm{NI}}(t+1)=P_{i}^{\mathrm{NI}}$. Near the outbreak threshold of epidemic spreading, the probability of an active node $i$ to be infected is approximately equal to zero, $P_{i}^{I} \longrightarrow 0$. Therefore, we can obtain

$$
q_{i} \approx 1-m_{2} \beta\left(b_{i} \rho^{I}+\theta_{b}^{I}\right),
$$

where $\theta_{b}^{I}=\sum_{j} b_{j} P_{j}^{I} / N$. Next, we give a proof to deduce the theoretical outbreak threshold $\beta_{c}$ of epidemic spreading.

Proof. By ignoring the higher-order items, we simplify equations (3)-(5) as

$$
\begin{gathered}
P_{i}^{\mathrm{RS}}=P_{i}^{\mathrm{RS}}\left[\delta q_{i}+(1-\delta) q_{i}\right]+P_{i}^{\mathrm{NI}}\left[\left(1-r_{i}\right) \mu^{R}+\mu^{N} r_{i}\right] \\
+P_{i}^{\mathrm{RI}}\left[(1-\delta) \mu^{R}+\delta \mu^{N}\right], \\
P_{i}^{\mathrm{NI}}=P_{i}^{\mathrm{NI}}\left(1-\mu^{N}\right) r_{i}+P_{i}^{\mathrm{RI}} \delta\left(1-\mu^{N}\right), \\
P_{i}^{\mathrm{RI}}=P_{i}^{\mathrm{NI}}\left(1-\mu^{R}\right)\left(1-r_{i}\right)+P_{i}^{\mathrm{RI}}(1-\delta)\left(1-\mu^{R}\right) .
\end{gathered}
$$

It is easy to find that the combination of equations (8) and (9) equals equation (7). By substituting equation (8) into equation (7), consequently, we can rewrite equation (7) as follows:

$$
P_{i}^{\mathrm{RS}}\left(1-q_{i}\right)=\mu^{R} P_{i}^{I}+\left(\mu^{N}-\mu^{R}\right) \frac{P_{i}^{\mathrm{NI}}}{1-\mu^{N}} .
$$

Note that $P_{i}^{\mathrm{RS}}=1-P_{i}^{\mathrm{NI}}-P_{i}^{\mathrm{RI}}$, and with ignoring higherorder items, the part $P_{i}^{\mathrm{RS}}\left(1-q_{i}\right)$ can be simplified as $(1-$ $\left.P_{i}^{\mathrm{RI}}\right)\left(1-q_{i}\right)$. Then, considering $\xi \longrightarrow 0\left(\right.$ i.e., $\left.\mu^{N} \ll \mu^{R}<1\right)$ and $P_{i}^{\mathrm{NI}}<P_{i}^{I} \ll 1$, we simplify equation (10) as follows:

$$
\mu^{R} P_{i}^{I}=\left(1-P_{i}^{\mathrm{RI}}\right)\left(1-q_{i}\right)
$$




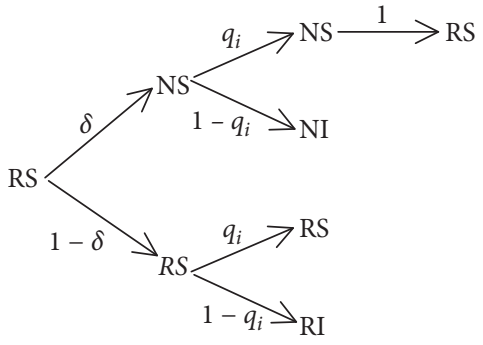

(a)

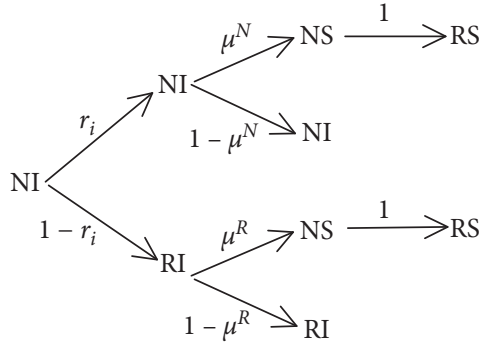

(b)

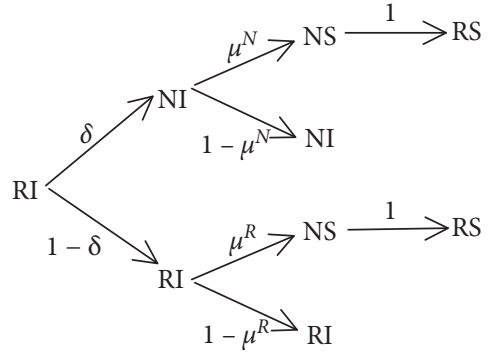

(c)

Figure 2: Transition probability trees of three types of nodes. They describe the transient changes of nodes' states at each time. The definitions of parameters can be found in Table 1.

Then, we note that $\theta_{b^{2}}^{\mathrm{RI}}=\sum_{j} b_{j}^{2} P_{j}^{\mathrm{RI}} / N$. Multiplying $b_{i}$ on both sides of equation (11) and taking average over all nodes, we obtain a similar expression of $\theta_{b}^{l}$ as

$$
\mu^{R} \theta_{b}^{I}=\left(1-\theta_{b}^{\mathrm{RI}}\right)\left(1-q_{i}\right) .
$$
form

Next, we consider equations (11) and (12) as a matrix

$$
\frac{\mu^{R}}{m_{2} \beta}\left[\begin{array}{c}
\rho^{I} \\
\theta_{b}^{I}
\end{array}\right]=\left[\begin{array}{cc}
\langle b\rangle-\theta_{b}^{\mathrm{RI}} & 1-\rho^{\mathrm{RI}} \\
\left\langle b^{2}\right\rangle-\theta_{b^{2}}^{\mathrm{RI}} & \langle b\rangle-\theta_{b}^{\mathrm{RI}}
\end{array}\right]\left[\begin{array}{c}
\rho^{I} \\
\theta_{b}^{I}
\end{array}\right],
$$

$\beta_{c}$ is the minimum value of $\beta$ that satisfies equation (13). And with $\left[\begin{array}{c}\rho^{I} \\ \theta_{b}^{I}\end{array}\right] \neq 0$, we can easily solve the critical point with iteration method through the matrix

$$
\beta_{c}=\frac{\mu^{R}}{m_{2}}\left\{\sqrt{\left(\left\langle b^{2}\right\rangle-\theta_{b^{2}}^{\mathrm{RI}}\right)\left(1-\rho^{\mathrm{RI}}\right)}+\langle b\rangle-\theta_{b}^{\mathrm{RI}}\right\}^{-1} .
$$

When there is only single layer in which only the epidemic spreads, the outbreak threshold of epidemic spreading could be solved by setting $\theta_{b}^{\mathrm{RI}}=0, \theta_{b^{2}}^{\mathrm{RI}}=0$, and $\rho^{\mathrm{RI}}=0$,

$$
\beta_{c}=\frac{\mu}{m_{2} \sqrt{\left\langle b^{2}\right\rangle+\langle b\rangle}} .
$$

\section{Results}

To validate our MMCA based theoretical analysis, we run numerical experiments by the Monte Carlo simulations (MC) and compare the experimental results with theoretical ones regarding different parameters. We construct the coupled network with 1,000 nodes by two AD models. Considering the identical social networks and referring to [36], we set the exponents of probability distributions of activity potential with $\gamma_{1}=\gamma_{2}=2.5$, the contact capacities of active nodes with $m_{1}=m_{2}=5$, and the rescaling factors with $\eta_{1}=\eta_{2}=1000$. The values of the rescaling factor do not affect the following results, but the activity heterogeneity and contact capacity have a significant influence, both of which are verified by the following experimental results. Note that the values of these parameters do not change unless they are chosen as the variables. We represent the final fraction of infected nodes $\rho_{I}$ and the fraction of noresource nodes $\rho_{N}$ in the stable states, which characterize the coupled dynamic processes of resource diffusion and epidemic spreading. Initially, we set a small fraction of infected nodes with $\rho_{I}=0.08$. Meanwhile, to highlight the crucial influence of individual resources on the recovery of disease, we herein set $\xi=0$ (i.e., $\mu^{N}=0$ and $\mu=\mu^{R}$ ) that means the infected nodes without resource cannot recover. In the following, we investigate the influences of a number of parameters involved with the RNR-SIS dynamic model and AD network model on the coupled dynamic processes by performing hundreds of MC numerical simulations and obtain a number of numerical results.

Firstly, we investigate the influence of $\beta$ on the coupled dynamic processes. In Figure 3, it can be found that the changes of $\rho_{I}$ and $\rho_{N}$ with $\beta$ indicate that there exists a phase transition of $\rho_{I}$ and $\rho_{N}$ when $\beta$ is larger than the critical points, and the critical points of $\rho_{I}$ and $\rho_{N}$ can be identified by the peak of variability $\chi$ calculated as [50]

$$
\chi=\omega \frac{\left\langle\rho^{2}\right\rangle-\langle\rho\rangle^{2}}{\langle\rho\rangle} .
$$

Note that, in Figure 3(b), we multiply $\omega=1000$ to identify the peak more clearly, which does not change the threshold value. For $\rho_{I}$, its critical point $\beta_{c}=0.12$ approximately equals the theoretical outbreak threshold of epidemic spreading by solving equation (14). Moreover, as shown in Figure 3(a), the numerical results of $\rho_{I}$ and $\rho_{N}$ are in good agreement with the theoretical ones obtained by solving equations (1)-(5) (the same in the following figures), which verifies the validation of MMCA based dynamic framework of the coupled model.

Then, we investigate the influences of $\delta$ and $\lambda$ on the coupled dynamic processes, which mainly imply how individual resource diffusion affects the epidemic spreading. Figure 4 shows the changes of $\rho_{I}$ and $\rho_{N}$ with $\delta$ and $\lambda$, respectively. Specifically, both $\rho_{I}$ and $\rho_{N}$ increase with $\delta$ in Figure 4(a) but decrease with $\lambda$ in Figure 4(b). And, the increasing (or decreasing) trend as a function of $\delta$ (or $\lambda$ ) is significantly different between resource diffusion and epidemic spreading. Obviously, $\delta$ and $\lambda$ have a greater influence on resource diffusion than epidemic spreading. More interestingly, a smaller $\lambda(\simeq 0.2)$ can dramatically reduce $\rho_{I}$, 


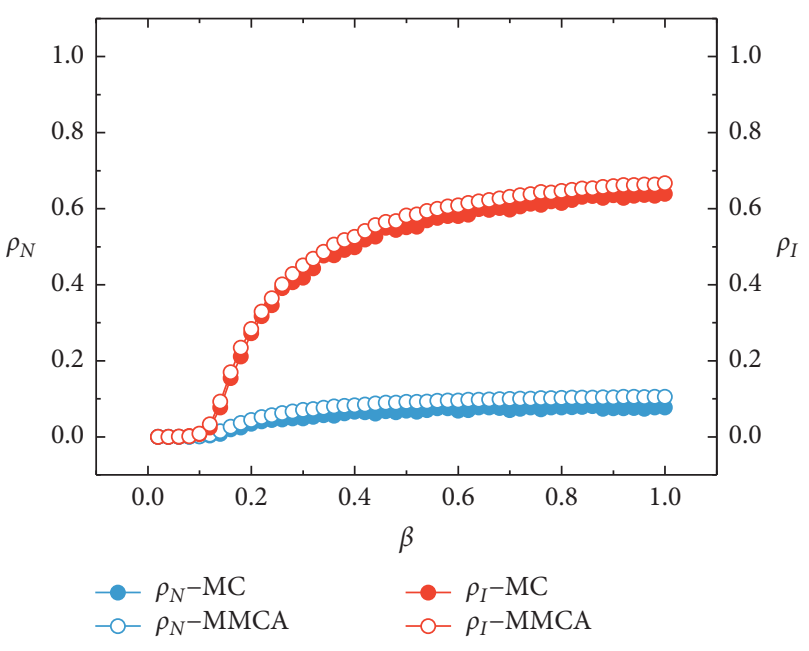

(a)

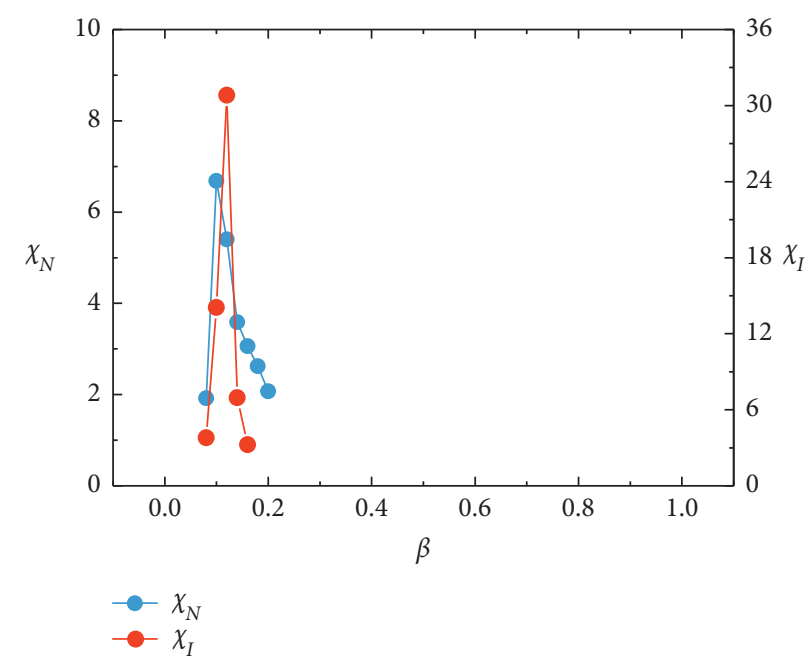

(b)

FIGURE 3: (a) The final fractions $\rho_{I}$ and $\rho_{N}$ of the infected nodes and the noresource nodes as a function of epidemic transmission rate $\beta$. The blue circle indicates $\rho_{N}$ and the red circle indicates $\rho_{I}$. For both $\rho_{I}$ and $\rho_{N}$, we present the results of numerical simulation and MMCA approach in full and open circles, respectively, which are in good agreement with each other. (b) The variability $\chi$ of $\rho_{I}$ and $\rho_{N}$ as a function of $\beta$. We can determine the critical points of $\rho_{I}$ and $\rho_{N}$ by the peak of variability and obtain the outbreak threshold of epidemic spreading $\beta_{c}=0.12$. The other fixed parameters are $\delta=0.10, \lambda=0.64, \mu=0.48$.

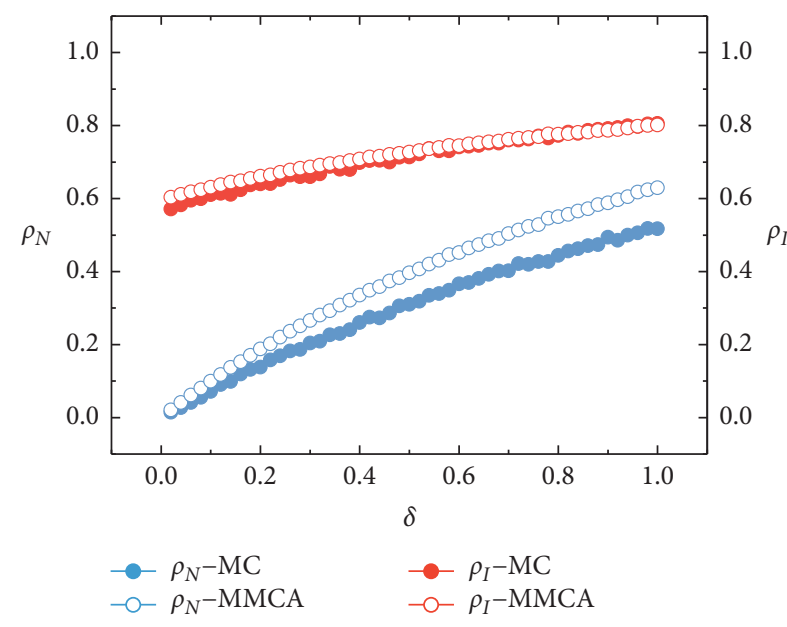

(a)

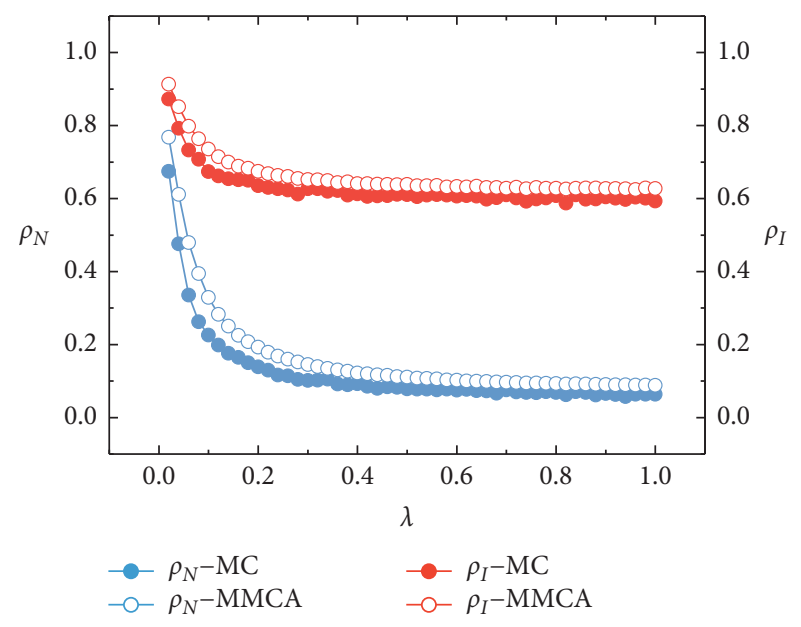

(b)

FIgURE 4: The final fractions $\rho_{I}$ and $\rho_{N}$ of the infected nodes and the noresource nodes as a function of the resource loss rate $\delta$ (a) and the resource diffusion rate $\lambda(\mathrm{b})$. The symbols are the same as in Figure 3. The results show that both $\rho_{I}$ and $\rho_{N}$ increase with $\delta$ but decrease with $\lambda$. And, a smaller $\lambda(\sim 0.2)$ can dramatically reduce $\rho_{I}$, indicating the diffusion of individual resources is of importance for suppressing the spread of disease. The other fixed parameters are $\beta=0.70, \mu=0.48$.

which, to some extent, suggests the diffusion of individual resources is of importance for suppressing the spread of disease.

We have investigated the influence of single parameter (i.e., $\delta, \lambda$, and $\beta$ ) on the coupled dynamic process. As the dynamic process of epidemic spreading is more important, we need the comprehensively synergistic influences of these parameters on the epidemic spreading and its outbreak threshold. Figure 5 shows the heat map of $\rho_{I}$ in respect to a pair of $\delta$ (or $\lambda$ ) and $\beta$. We can find that, in Figure 5(a), the theoretical outbreak threshold $\beta_{c}$ weakly decreases with $\delta$, and in Figure 5(b), it quickly increases then barely changes with $\lambda$ which indicates that the resource diffusion suppresses the outbreak threshold with a lower resource loss rate and larger resource diffusion rate. Meanwhile, when the epidemic outbreaks (i.e., $\beta>\beta_{c}$ ), it can be seen that smaller $\delta$ and larger $\lambda$ can more effectively suppress the spread of disease, confirming the conclusion found in Figure 4. We also calculate the (simulated) outbreak threshold of $\lambda$ obtained by the variability measurement in equation (16) based on the Monte Carlo simulations of numerical experiments, which are shown with the transverse curve in Figure 5(b). 


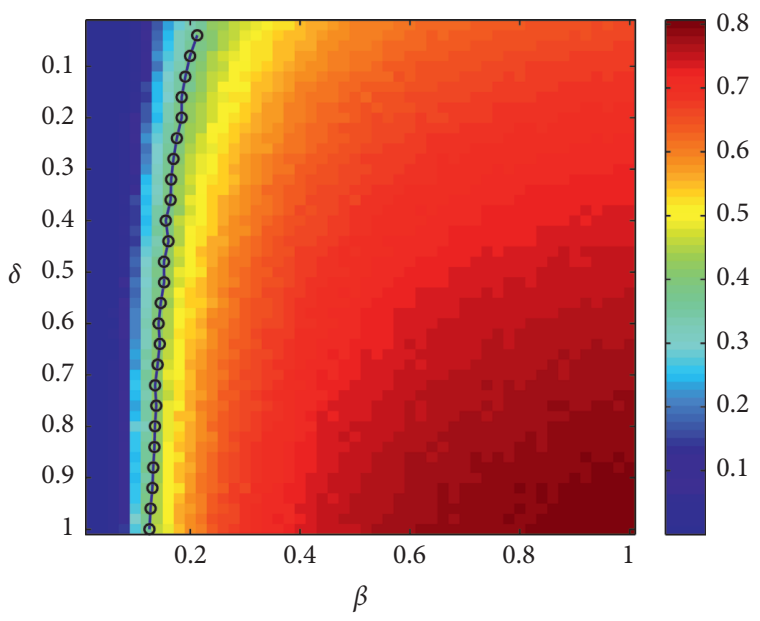

(a)

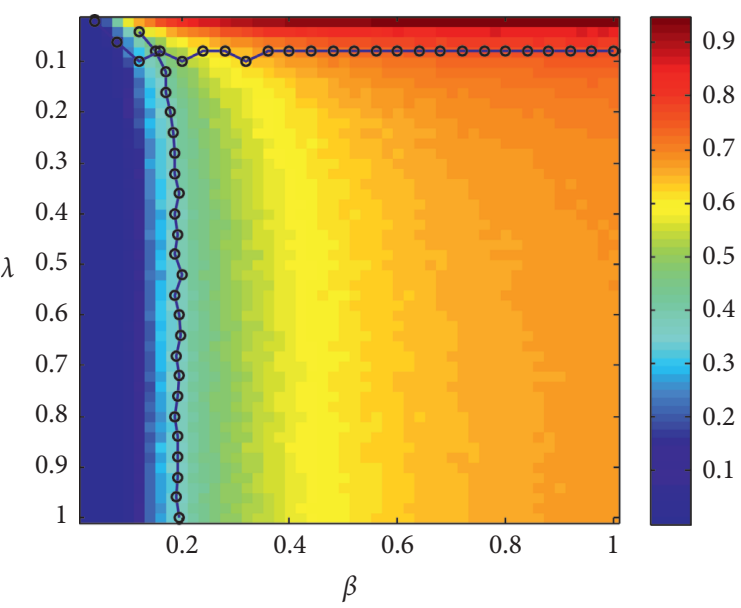

(b)

Figure 5: The heat maps of $\rho_{I}$ as a function of a pair of $\delta$ and $\beta$ (a) and $\lambda$ and $\beta$ (b). The longitudinal curve in Figures 5(a) and 5(b) indicates the theoretical outbreak thresholds of $\beta$ obtained by solving equation (13), which is the same as those in Figure 7 . And, the transverse curve in Figure 5(b) indicates the simulated outbreak thresholds of $\lambda$ obtained by the variability measurement, which affects the outbreak of epidemic. It trivially fluctuates only when $\beta$ is less than 0.2 . These two subfigures clearly show that $\delta$ and $\lambda$ affect the outbreak thresholds of each other and effectively affect the outbreak of the epidemic, which suggests that resource diffusion can play an important role in the control of epidemic spreading. The other fixed parameters are $\lambda=0.64$ for (a), $\delta=0.10$ for (b), $\mu=0.48$.

When $\lambda$ is less than the simulated outbreak threshold, the epidemic will outbreak even if $\beta$ is very small. We also note that $\lambda_{c}$ (i.e., the transverse curve) fluctuates only when $\beta$ is less than 0.2. A smaller $\beta$ results in a smaller threshold of resource diffusion rate that a lower rate of epidemic spreading can reduce the risk of an epidemic outbreak caused by a low rate of resource diffusion. However, a smaller $\lambda$ results in a smaller $\beta_{c}$ making it easy for an epidemic outbreak. These results suggest that the diffusive dynamics of individual resources can affect both the outbreak threshold and the spread of disease.

Next, we make efforts to investigate the influences of time-varying network structure mainly including activity heterogeneity and contact capacity of nodes in two AD networks on the coupled dynamic process. Figures 6(a) and 6(b) show that $\gamma_{1}$ and $m_{1}$ in the resource layer, respectively, impact $\rho_{I}$ and $\rho_{N}$. They clearly show both $\rho_{I}$ and $\rho_{N}$ weekly increase with $\gamma_{1}$ in Figure 6(a) and decrease with $m_{1}$ in Figure $6(\mathrm{~b})$. The finding suggests that stronger activity heterogeneity (corresponding to smaller $\gamma_{1}$ ) and larger contact capacity effectively promote resource diffusion (corresponding to smaller $\rho_{N}$ ) but suppress epidemic spreading (corresponding to smaller $\rho_{I}$ ). Note that, in comparison with the epidemic spreading, $\gamma_{1}$ and $m_{1}$ have more influence on the dynamic process of resource diffusion.

In contrast, we present that $\gamma_{2}$ and $m_{2}$ in the epidemic layer, respectively, impact $\rho_{I}$ and $\rho_{N}$ in Figures 6(c) and 6(d). They clearly shows that both $\rho_{I}$ and $\rho_{N}$ weakly decrease with $\gamma_{2}$ in Figure 6(c) and increase with $m_{2}$ in Figure 6(d). These results are the opposite to the ones found in Figures 6(a) and 6(b), which suggest that stronger activity heterogeneity (corresponding to smaller $\gamma_{2}$ ) and larger contact capacity cause more resource depletion (corresponding to larger $\rho_{N}$ ) and thus promote epidemic spreading (corresponding to larger $\left.\rho_{I}\right)$. Also, in comparison with the resource diffusion, we note $\gamma_{2}$ and $m_{2}$ having more influences on the dynamic process of epidemic spreading.

The following results also focus on the synergistic influences of $\gamma_{i}, m_{i}$, and $\beta$ on the epidemic spreading and its outbreak threshold. It is clearly shown that, in Figure 7(a), the outbreak threshold is almost uncorrelated with $\gamma_{1}$ and, in Figure 7(b), it weakly increases and then barely changes with $m_{1}$. Meanwhile, for $\beta>\beta_{c}$, we show that the spread of disease is obviously suppressed by more heterogeneous activity potential and larger contact capacity of nodes in the resource layer, which confirms the conclusion found in Figures 6(a) and $6(\mathrm{~b})$. These results indicate that the dynamic social relationships of individuals represented by the $\mathrm{AD}$ network model in the resource layer cannot inhibit the outbreak of the disease but can effectively suppress the spread of disease.

In contrast, in Figures 7(c) and 7(d), we can find that the outbreak threshold is strongly affected by $\gamma_{2}$ and $m_{2}$. More specifically, when the activity potentials of individuals in the epidemic layer become more heterogeneous (corresponding to smaller $\gamma_{2}$ ), the outbreak thresholds dramatically decrease. When the contact capacities of individuals in the epidemic layer are much larger, the outbreak thresholds also dramatically decrease. Meanwhile, for $\beta>\beta_{c}$, we find that stronger activity heterogeneity and larger contact capacity of individuals in the epidemic layer significantly promote the spread of disease, which also confirms the conclusion found in Figures 6(c) and 6(d). Besides, combining with the results in Figures 7(b) and 7(d), it can be seen that, in respect to each 


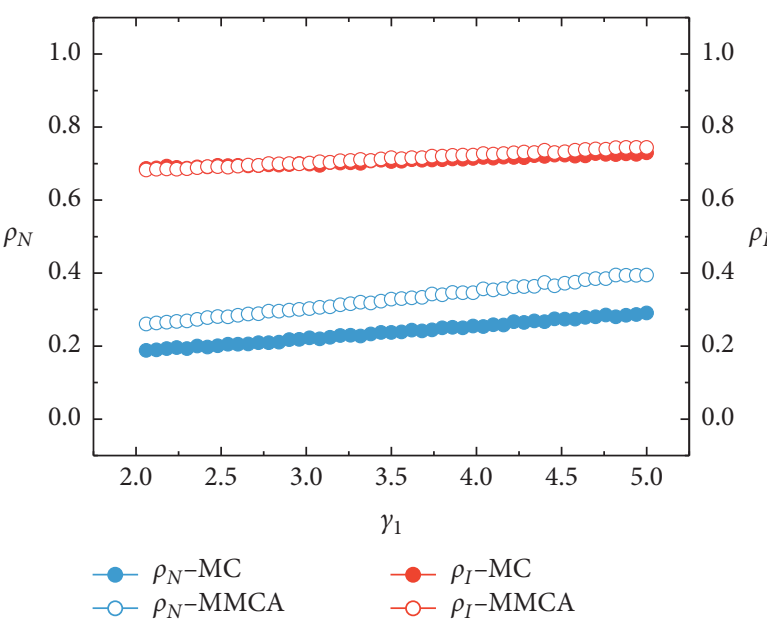

(a)

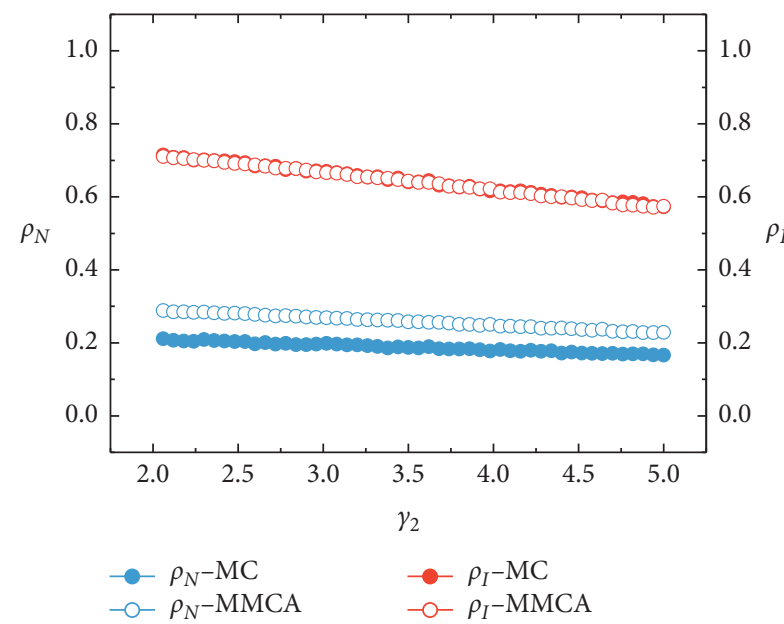

(c)

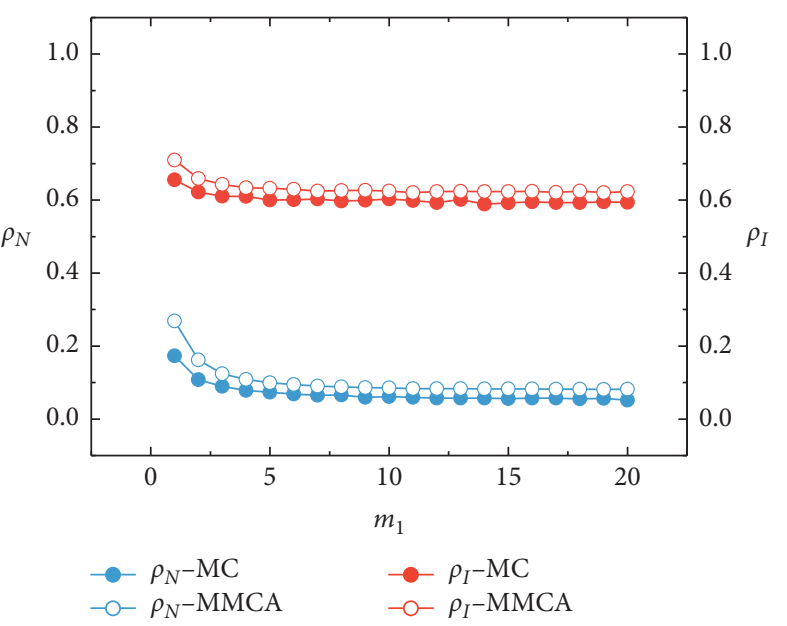

(b)

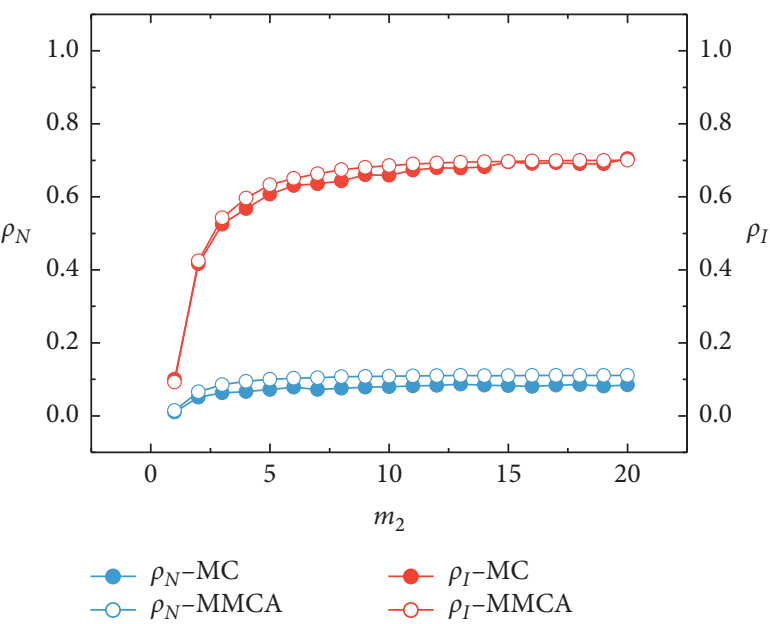

(d)

Figure 6: The final fractions $\rho_{I}$ and $\rho_{N}$ of the infected nodes and the noresource nodes as a function of activity potential $\gamma_{i}$ and the contact capacity $m_{i}$. The symbols are the same as those in Figure 3. The upper panel presents that $\rho_{I}$ and $\rho_{N}$ changes with $\gamma_{1}$ (a) and $m_{1}$ (b) in the resource layer, which shows that stronger activity heterogeneity and larger contact capacity of individuals in the resource layer can effectively promote resource diffusion but suppress epidemic spreading. The lower panel presents that $\rho_{I}$ and $\rho_{N}$ changes with $\gamma_{2}$ (a) and $m_{2}$ (b) in the epidemic layer, which suggests that these two characters of individuals in the epidemic layer cause more resource depletion and thus promote epidemic spreading. The other fixed parameters are $\delta=0.10, \lambda=0.64, \beta=0.70, \mu=0.48$, and $m_{1}=5$ for (a), (c), and (d); $m_{2}=5$ for (a), (b), and (c); $\gamma_{1}=2.5$ for (b), (c), and (d); and $\gamma_{2}=2.5$ for (a), (b), and (d).

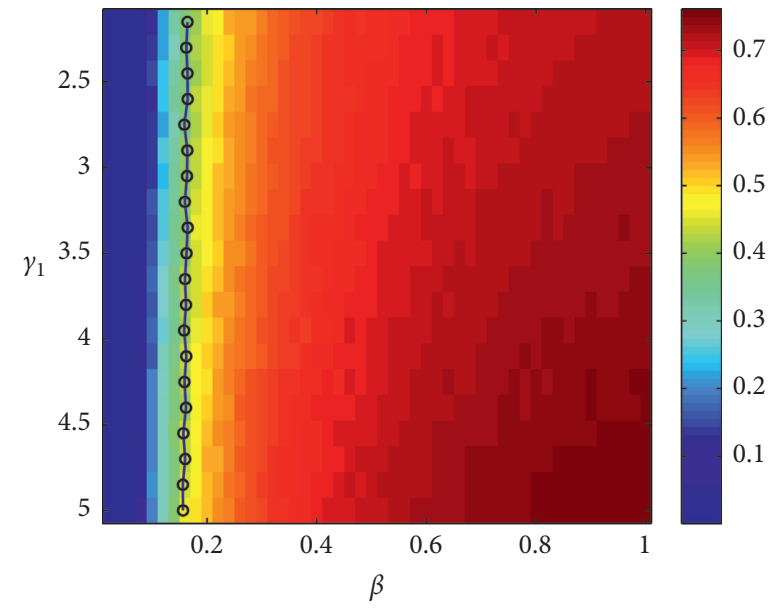

(a)

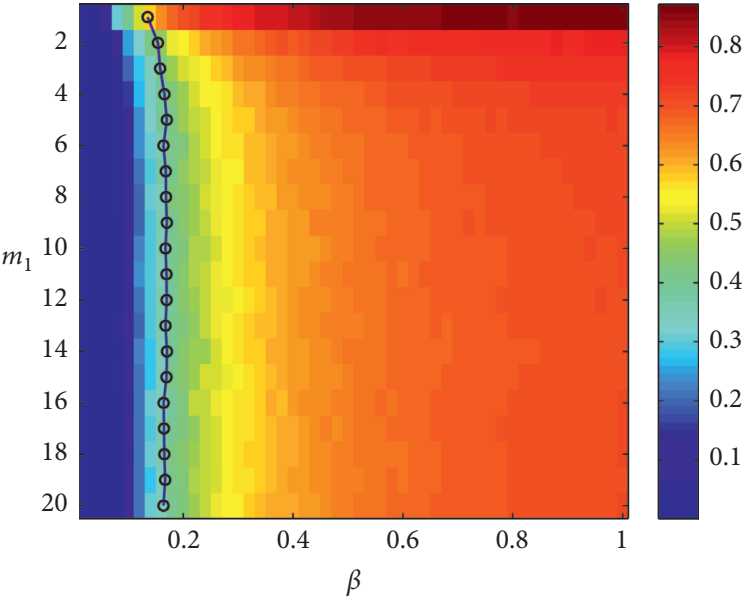

(b)

Figure 7: Continued. 


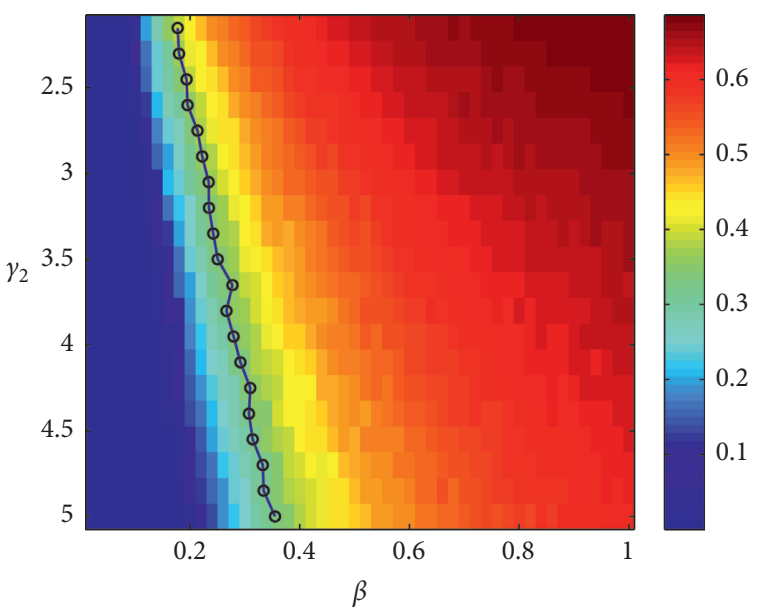

(c)

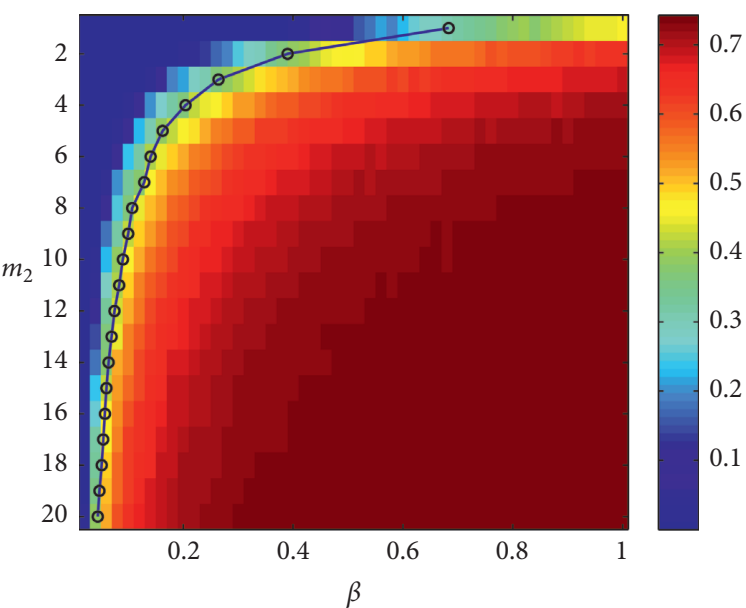

(d)

Figure 7: The heat maps of $\rho_{I}$ as a function of a pair of $\gamma_{i}$ and $\beta(\mathrm{a}-\mathrm{c})$ and $m_{i}$ and $\beta(\mathrm{b}-\mathrm{d})$. (a,b) $\gamma_{1}$ and $m_{1}$ are approximately uncorrelated with the outbreak threshold and effectively affect the spread of disease, which suggests that the dynamic social relationships of individuals in the resource layer cannot inhibit the outbreak of the disease but can effectively suppress the spreading of disease. (c, d) $\gamma_{2}$ and $m_{2}$ significantly affect the outbreak threshold and the spread of disease, which suggests that the dynamic physical contacts of individuals in the epidemic layer have strong influences on the dynamic process of epidemic spreading. The other fixed parameters are $\delta=0.10, \lambda=0.64$, $\mu=0.48$, and $m_{1}=5$ for (a), (c), and (d); $m_{2}=5$ for (a), (b), and (c); $\gamma_{1}=2.5$ for (b), (c), and (d); and $\gamma_{2}=2.5$ for (a), (b), and (d).

$\beta\left(>\beta_{c}\right)$, the contact capacity of individuals in both the resource and epidemic layers has a limited influence on the spread of disease.

\section{Conclusion}

In conclusion, the present work has investigated the coupled dynamic processes of resource diffusion and epidemic spreading via the proposed RNR-SIS dynamic model on time-varying multiplex networks. We perform the investigations by assuming a good abstraction from a real scenario, where the diseases spread on a physical contact network, the individual resources diffuse on an online social network, and they dynamically interact with each other. Considering such two networks are temporal due to the time-varying physical contacts or social relationships of individuals, we first construct the time-varying two-layer network by using two $\mathrm{AD}$ network models with different parameters. The AD model encodes the connectivity pattern (i.e., the dynamic process of changing physical contact or social relationship) of individuals in the distribution of activity potential following a power-law function empirically measured in realworld networks. This function allows the definition of a simple dynamic process based on the nodal activity level, providing a time-dependent description of the connectivity pattern.

Then, to explore the full-phase diagram of the coupled RNR-SIS dynamics on the time-varying two-layer network, we develop a probabilistic framework of the intralayer and interlayer dynamic processes of the coupled model by extending MMCA to time-varying multiplex networks. Based on the theoretical analysis of the probabilistic framework, we obtain the expressions of the fractions of infected nodes and noresource nodes and the outbreak threshold. Monte Carlo simulations are essential to verify the theoretical analysis. Comparing with extensive Monte Carlo simulations of the same system. The approximation using MMCA has an accuracy of up to $1.93 \%$ error for the prediction of the epidemic incidence and $21.79 \%$ error for the outbreak threshold of $\beta$. The principal reason for the large error in $\beta_{c}$ is that we neglect some higher-order terms and other related terms. The approximate agreement between theoretical and numerical results indicates the validation of the determined probabilistic framework. Meanwhile, these results show the trivially asymmetric interactions between the coupled dynamic process of resource diffusion and epidemic spreading. That is, the spread of disease is effectively suppressed by the resource diffusion with a lower resource loss rate and larger resource diffusion rate, but the diffusion of individual resources is trivially affected by the epidemic spreading even with a very larger disease transmission rate. Besides, we note that when epidemic outbreaks, the resource diffusion rate has a limited influence on the outbreak threshold and the fraction of infected nodes and also the disease transmission rate affects the threshold of resource diffusion rate limitedly around the threshold of the disease transmission rate.

Next, considering the influence of time-varying twolayer network structure on the coupled dynamics of resource diffusion and epidemic spreading, we mainly focus on how the activity potential and contact capacity of individuals in the resource and epidemic layers affect the final fraction of infected nodes and noresource nodes and the outbreak threshold. The theoretical and numerical results suggest that stronger activity heterogeneity and larger contact capacity of individuals in the resource layer can suppress the spread of disease because they promote the diffusion of individual resources. In contrast, these two characters of individuals in 
the epidemic layer tend to mitigate the diffusion of individual resources and thus increase epidemic prevalence. Meanwhile, we note that the outbreak threshold is nearly independent of the time-varying network structure in the resource layer, suggesting the resource diffusion of individuals cannot inhibit the outbreak of disease but only can effectively suppress the spreading of disease. Besides, when the epidemic outbreaks, the contact capacity of individuals in both the resource and epidemic layer has a finite influence on the final fraction of infected nodes, suggesting that increasing contact capacity above a very large value makes no sense at all.

Through the conclusion mentioned above, it can be seen that the diffusion of individual resources interacts closely with the dynamic process of epidemic spreading. On the one hand, the quicker diffusion of individual resources can effectively suppress epidemic spreading. However, only considering the dynamics of resource diffusion, we easily waste the individual resources due to the limited influence of the resource diffusion rate. On the other hand, we turn to analyze how the time-varying network structure affects the coupled dynamic process. When the epidemic outbreaks, individuals with larger activity potential and contact capacity have a high risk of getting infected but also are able to quickly obtain the resources of the neighbors. An extreme scenario is that the lack of individual resources might happen if the epidemic deteriorates, and then it entered a vicious circle. Thus, the obtained conclusion may be useful to provide methods of controlling large-scale epidemic prevalence, such as strengthening resource diffusion, reducing resource loss, speeding up of individuals' activities, and enlarging the contact capacity in social networks. Finally, the proposed coupled resource-epidemic model and analytical approach may be instructive to multiplex spreading models in time-varying multilayer networks.

\section{Data Availability}

Correspondence and requests for data should be addressed to S-M. C. (e-mail: shimincai@uestc.edu.cn).

\section{Conflicts of Interest}

The authors declare that there are no conflicts of interest regarding the publication of this paper.

\section{Acknowledgments}

This work was supported by National Natural Science Foundation of China (Grant nos. 61673086, 11975099, and 11575041), the Science and Technology Department of Sichuan Province (Grant no. 2020YFS0007), the Chendu Science and Technology Agency (Grant no. 2020-YF0500073-SN), and the Science Promotion Programme of UESTC, China (no. Y03111023901014006).

\section{References}

[1] J. Radcliffe and N. T. J. Bailey, "The mathematical theory of infectious diseases and its applications. 2nd edition," Applied Statistics, vol. 26, no. 1, pp. 85-87, 1977.

[2] D. J. Daley and J. Gani, Epidemic Modelling: An Introduction, Cambridge University Press, Cambridge, UK, 2001.

[3] R. M. Anderson, B. Anderson, and R. M. May, Infectious Diseases of Humans: Dynamics and Control, Oxford University Press, Oxford, UK, 1991.

[4] S. Boccaletti, G. Bianconi, R. Criado et al., "The structure and dynamics of multilayer networks," Physics Reports, vol. 544, no. 1, pp. 1-122, 2014.

[5] R. Pastor-Satorras, C. Castellano, P. Van Mieghem, and A. Vespignani, "Epidemic processes in complex networks," Reviews of Modern Physics, vol. 87, no. 3, p. 925, 2015.

[6] W. Wang, Q.-H. Liu, J. Liang, Y. Hu, and T. Zhou, "Coevolution spreading in complex networks," Physics Reports, vol. 820, pp. 1-51, 2019.

[7] D. J. Earn, P. Rohani, B. M. Bolker, and B. T. Grenfell, “A simple model for complex dynamical transitions in epidemics," Science, vol. 287, no. 5453, pp. 667-670, 2000.

[8] M. Martcheva and S. S. Pilyugin, "An epidemic model structured by host immunity," Journal of Biological Systems, vol. 14, no. 2, pp. 185-203, 2006.

[9] G. Hartvigsen, J. M. Dresch, A. L. Zielinski, A. J. Macula, and C. C. Leary, "Network structure, and vaccination strategy and effort interact to affect the dynamics of influenza epidemics," Journal of Theoretical Biology, vol. 246, no. 2, pp. 205-213, 2007.

[10] J. O. Hill, J. C. Peters, and H. R. Wyatt, "The role of public policy in treating the epidemic of global obesity," Clinical Pharmacology \& Therapeutics, vol. 81, no. 5, pp. 772-775, 2007.

[11] H.-F. Zhang, P.-P. Shu, Z. Wang, M. Tang, and M. Small, "Preferential imitation can invalidate targeted subsidy policies on seasonal-influenza diseases," Applied Mathematics and Computation, vol. 294, pp. 332-342, 2017.

[12] Z. Wang, Y. Moreno, S. Boccaletti, and M. Perc, "Vaccination and epidemics in networked populations-An introduction," Chaos, Solitons \& Fractals, vol. 103, pp. 177-183, 2017.

[13] S. Funk, E. Gilad, C. Watkins, and V. A. A. Jansen, "The spread of awareness and its impact on epidemic outbreaks," Proceedings of the National Academy of Sciences, vol. 106, no. 16, pp. 6872-6877, 2009.

[14] S. Samanta, S. Rana, A. Sharma, A. K. Misra, and J. Chattopadhyay, "Effect of awareness programs by media on the epidemic outbreaks: a mathematical model," Applied Mathematics and Computation, vol. 219, no. 12, pp. 69656977, 2013.

[15] A. Rizzo, M. Frasca, and M. Porfiri, "Effect of individual behavior on epidemic spreading in activity-driven networks," Physical Review E, vol. 90, no. 4, Article ID 042801, 2014.

[16] K. Paarporn, C. Eksin, J. S. Weitz, and J. S. Shamma, "Networked sis epidemics with awareness," IEEE Transactions on Computational Social Systems, vol. 4, no. 3, pp. 93-103, 2017.

[17] C. Zheng, C. Xia, Q. Guo, and M. Dehmer, "Interplay between sir-based disease spreading and awareness diffusion on multiplex networks," Journal of Parallel and Distributed Computing, vol. 115, pp. 20-28, 2018. 
[18] C. Xia, Z. Wang, C. Zheng et al., "A new coupled diseaseawareness spreading model with mass media on multiplex networks," Information Sciences, vol. 471, pp. 185-200, 2019.

[19] H. Yang, C. Gu, M. Tang, S.-M. Cai, and Y.-C. Lai, "Suppression of epidemic spreading in time-varying multiplex networks," Applied Mathematical Modelling, vol. 75, pp. 806-818, 2019.

[20] M. L. Brandeau, G. S. Zaric, and A. Richter, "Resource allocation for control of infectious diseases in multiple independent populations: beyond cost-effectiveness analysis," Journal of Health Economics, vol. 22, no. 4, pp. 575-598, 2003.

[21] X. Chen, T. Zhou, L. Feng et al., "Nontrivial resource requirement in the early stage for containment of epidemics," Physical Review E, vol. 100, no. 3, Article ID 032310, 2019.

[22] N. J. Watkins, C. Nowzari, V. M. Preciado, and G. J. Pappas, "Optimal resource allocation for competitive spreading processes on bilayer networks," IEEE Transactions on Control of Network Systems, vol. 5, no. 1, pp. 298-307, 2016.

[23] X.-L. Chen, S.-M. Cai, M. Tang, W. Wang, T. Zhou, and P.-M. Hui, "Controlling epidemic outbreak based on local dynamic infectiousness on complex networks," Chaos: An Interdisciplinary Journal of Nonlinear Science, vol. 28, no. 12, Article ID 123105, 2018.

[24] X. Chen, R. Wang, T. Ming, S. Cai, H. E. Stanley, and L. A. Braunstein, "Suppressing epidemic spreading in multiplex networks with social-support," New Journal of Physics, vol. 20, no. 1, Article ID 013007, 2018.

[25] X. Chen, W. Wang, S. Cai, H. E. Stanley, and L. A. Braunstein, "Optimal resource diffusion for suppressing disease spreading in multiplex networks," Journal of Statistical Mechanics, vol. 2018, no. 5, Article ID 053501, 2018.

[26] X.-L. Chen, R.-J. Wang, C. Yang, and S.-M. Cai, "Hybrid resource allocation and its impact on the dynamics of disease spreading," Physica A: Statistical Mechanics and Its Applications, vol. 513, pp. 156-165, 2019.

[27] R. Wang, X. Chen, and S. Cai, "Local information based resource allocation model for disease suppressing on complex networks," Physica A: Statistical Mechanics and Its Applications, vol. 533, p. 121968, 2019.

[28] S. Bansal, J. Read, B. Pourbohloul, and L. A. Meyers, "The dynamic nature of contact networks in infectious disease epidemiology," Journal of Biological Dynamics, vol. 4, no. 5, pp. $478-489,2010$.

[29] E. Valdano, M. R. Fiorentin, C. Poletto, and V. Colizza, "Epidemic threshold in continuous-time evolving networks," Physical Review Letters, vol. 120, no. 6, pp. $068302.1-068302.6,2018$.

[30] J. Sun, L. Feng, J. Xie, X. Ma, D. Wang, and Y. Hu, "Revealing the predictability of intrinsic structure in complex networks," Nature Communications, vol. 11, no. 1, p. 574, 2020.

[31] R. K. Pan and J. Saramäki, "Path lengths, correlations, and centrality in temporal networks," Physical Review E, vol. 84, Article ID 016105, 2011.

[32] P.-B. Cui, W. Wang, S.-M. Cai, T. Zhou, and Y.-C. Lai, "Close and ordinary social contacts: how important are they in promoting large-scale contagion?" Physical Review E, vol. 98, no. 5, Article ID 052311, 2018.

[33] X.-H. Chen, S.-M. Cai, W. Wang, M. Tang, and H. E. Stanley, "Predicting epidemic threshold of correlated networks: a comparison of methods," Physica A: Statistical Mechanics and Its Applications, vol. 505, pp. 500-511, 2018.

[34] P. Block, M. Hoffman, I. J. Raabe et al., "Social network-based distancing strategies to flatten the covid-19 curve in a post- lockdown world," Nature Human Behaviour, vol. 4, pp. 588-596, 2020.

[35] J. S. Weitz, S. J. Beckett, A. R. Coenen et al., "Modeling shield immunity to reduce covid-19 epidemic spread," Nature Medicine, vol. 26, pp. 849-854, 2020.

[36] N. Perra, B. Gonçalves, R. Pastor-Satorras, and A. Vespignani, "Activity driven modeling of time varying networks," Scientific Reports, vol. 2, p. 469, 2012.

[37] B. Ribeiro, N. Perra, and A. Baronchelli, "Quantifying the effect of temporal resolution on time-varying networks," Scientific Reports, vol. 3, p. 3006, 2013.

[38] M.-X. Liu, W. Wang, Y. Liu, M. Tang, S.-M. Cai, and H.-F. Zhang, "Social contagions on time-varying community networks," Physical Review E, vol. 95, no. 5, Article ID 052306, 2017.

[39] A. Moinet, R. Pastor-Satorras, and A. Barrat, "Effect of risk perception on epidemic spreading in temporal networks," Physical Review E, vol. 97, no. 1, Article ID 012313, 2018.

[40] M. Newman, "Spread of epidemic disease on networks," Physical Review. E, Statistical, Nonlinear, and Soft Matter Physics, vol. 66, Article ID 016128, 082002.

[41] S. Gómez, J. Gómez-Gardenes, Y. Moreno, and A. Arenas, "Nonperturbative heterogeneous mean-field approach to epidemic spreading in complex networks," Physical Review E, vol. 84, no. 3, Article ID 036105, 2011.

[42] W. Wang, M. Tang, H. E. Stanley, and L. A. Braunstein, "Unification of theoretical approaches for epidemic spreading on complex networks," Reports on Progress in Physics, vol. 80, no. 3, Article ID 036603, 2017.

[43] S. Gómez, A. Arenas, J. Borge-Holthoefer, S. Meloni, and Y. Moreno, "Discrete-time Markov chain approach to contact-based disease spreading in complex networks," $E P L$ (Europhysics Letters), vol. 89, no. 3, p. 38009, 2010.

[44] C. Granell, S. Gómez, and A. Arenas, "Dynamical interplay between awareness and epidemic spreading in multiplex networks," Physical Review Letters, vol. 111, no. 12, p. 128701, 2013.

[45] C. Granell, S. Gomez, and A. Arenas, "Competing spreading processes on multiplex networks: awareness and epidemics," Physical Review E, vol. 90, no. 1, Article ID 012808, 2014.

[46] J.-Q. Kan and H.-F. Zhang, "Effects of awareness diffusion and self-initiated awareness behavior on epidemic spreading-an approach based on multiplex networks," Communications in Nonlinear Science and Numerical Simulation, vol. 44, pp. 193-203, 2017.

[47] Z. Wang, Q. Guo, S. Sun, and C. Xia, "The impact of awareness diffusion on sir-like epidemics in multiplex networks," $A p$ plied Mathematics and Computation, vol. 349, pp. 134-147, 2019.

[48] Q. Guo, Y. Lei, X. Jiang, Y. Ma, G. Huo, and Z. Zheng, "Epidemic spreading with activity-driven awareness diffusion on multiplex network," Chaos, vol. 26, no. 4, Article ID 043110, 2016.

[49] Y.-H. Qin, Z.-D. Zhao, S.-M. Cai, L. Gao, and H. E. Stanley, "Dual-induced multifractality in online viewing activity," Chaos, vol. 28, no. 1, Article ID 013114, 2018.

[50] M. Catanzaro, M. Boguná, and R. Pastor-Satorras, "Generation of uncorrelated random scale-free networks," Physical Review E, vol. 71, no. 2, Article ID 027103, 2005. 